\title{
Interdisciplinary conversations in STEM education: can faculty understand each other better than their students do?
}

\author{
Jeffrey M. Rabin 1* (D), Adam Burgasser², Thomas J. Bussey ${ }^{3}$, John Eggers ${ }^{1}$, Stanley M. Lo ${ }^{4}$, Sherry Seethaler ${ }^{5}$, \\ Laura Stevens ${ }^{1}$ and Haim Weizman ${ }^{3}$
}

\begin{abstract}
Rate of change concepts from calculus are presented and applied rather differently in college mathematics, physics, biology, and chemistry classes. This is not simply a matter of pedagogical style but reflects real cultural differences between these disciplines. We describe the efforts of our interdisciplinary collaboration to understand and reconcile these differences as we designed and discussed instructional videos for students. We summarize our conversations about terminology, notation, functions, rates, units, and sign conventions across the disciplines. We present some strategies that enabled us to communicate effectively, resolve confusions, and reach shared understandings. Our work has implications for others involved in collaborative interdisciplinary projects and for STEM educators.

In theory, there's no difference between theory and practice. But in practice, there is.

- Benjamin Brewster. Also attributed to Yogi Berra.
\end{abstract}

Keywords: Interdisciplinarity, STEM education, Rate of change, Communication, Scientific collaboration

\section{Introduction}

In this paper, we describe the discussions of our interdisciplinary group of scientists and mathematicians during a multi-year curriculum project collaboration. Our goal is to highlight subtle differences between concepts that are nominally "the same" across multiple disciplines, the confusions that both students and experts can encounter about them, and the importance of STEM instructors being aware of them. Science instructors may expect that the mathematics their students learn in prerequisite math courses will be in a ready-to-use format adapted to its applications in science, but this may not be the case. Likewise, mathematics instructors may incorrectly assume that scientific applications of calculus will use the same conceptual structures, notation, and

\footnotetext{
*Correspondence: jrabin@ucsd.edu

'Department of Mathematics, University of California San Diego, La Jolla, CA, USA

Full list of author information is available at the end of the article
}

terminology presented in calculus texts. The more STEM educators know about such disciplinary cultural differences, the more they can help their students to anticipate confusions and make connections.

Since 2016, the authors have been engaged in an NSFfunded project to create a series of short instructional videos for college students of biology, chemistry, mathematics, and physics dealing with rate of change concepts from calculus. The videos, each about $7 \mathrm{~min}$ in length, deal with four core concepts (meaning of terms in differential equations, average versus instantaneous rate of change, moving among graphical representations, relationship between rates and accumulation) in the context of each of the four disciplines. We assume that students have already seen the topics in their courses, so the videos do not introduce them from scratch, but address important conceptual issues and common misconceptions across the four subject areas. The treatment of the examples in each discipline is faithful to that 
discipline, but the cross-cutting nature of the concepts should also be evident to students. The primary purpose is to help students connect the mathematics they learn in their mathematics classes with the same mathematics as applied in science.

Typically, one member of our team drafts a script (including visuals) for a particular video, the entire team reads it, and a critical discussion ensues. This is followed by script revision and iteration of the process. When the entire team is satisfied, filming and animation take place. A broad range of issues arise in the discussions. The situations, notation, terminology, and reasoning in the script should be faithful to the discipline, yet consistent or at least understandable across disciplines. Explanations should be scientifically accurate yet not so technical as to obscure the key ideas, and should build on students' existing knowledge. Emphasis should be on the rate of change concepts as they are actually used in each discipline.

Many design decisions relate to other aspects of pedagogy, including affective considerations. For example, student characters in the videos should be both believable and empowered, not simply confused and deferentially asking authority figures for information. While the present paper focuses on our discussions of specific mathematical content, another publication by our team (Seethaler et al., 2020) synthesizes a wide body of educational research relevant to informing video development more generally.

As we engaged in these discussions, we repeatedly found that each of our disciplines has its own conventions, implicit assumptions, representations, notations, canonical examples, and terminology. Distinctions that are considered important in one discipline may not be emphasized or even recognized in others. Concepts that are nominally "the same" may appear quite different across disciplines; we often found ourselves asking each other, "why would you do it that way?" It was surprisingly challenging for us to reconcile these differences for ourselves, let alone decide what sort of synthesis to present to students. Because our own interdisciplinary communication was more challenging than we expected, we added to our meetings an explicit focus on understanding the viewpoints of other disciplines and critically assessing our own disciplinary perspectives. We came to value this aspect of our discussions as much as the end goal of producing the videos. It broadened our own appreciation and understanding of these issues, suggested alternate pedagogical approaches, and gave us new respect and empathy for students struggling to connect what they learn in their mathematics and science classes.

An essential feature of our discussions was our willingness to ask and pursue "elementary" questions without (too much) embarrassment. Due to our comfort level with one another, and respect for our colleagues' disciplinary expertise, we were able to express confusions about even basic ideas in one another's fields and talk through them until we achieved some common understanding.

The content of our videos was informed by the extensive literature on the teaching and learning of rate of change concepts in both mathematics and science education. In particular, Thompson and collaborators have developed a coherent treatment of the measurement and representation of quantities, the mathematical description of covariation by functions, average and instantaneous rates of change as quantities, and the integral viewed as an accumulation function (Thompson, 1994a; Thompson, 1994b; Thompson, Byerley, \& Hatfield, 2013; Thompson \& Carlson, 2017; Thompson \& Silverman, 2008). Bain and coworkers have discussed student thinking about rate of change in the context of chemical kinetics (Bain \& Towns, 2016; Rodriguez, Bain, Towns, Elmgren, \& Ho, 2018). Seethaler, Czworkowski, and Wynn (2018) have investigated chemistry textbook treatments of rates of change. Jones has explored students' understanding of derivatives in real-world contexts (Jones, 2017) and has drawn implications from calculus education research for chemistry instruction (Jones, 2019). Other useful discussions of rates of change in applied contexts are Dray, Gire, Kustusch, Manogue, and Roundy (2019) and Herbert and Pierce (2011).

The limited literature we are aware of about interdisciplinary communication among STEM fields is split between collaborations having scientific research goals and those like ours having educational goals. Duncker (2001) describes a research collaboration between departments of chemical physics, materials science, philosophy of science, organic chemistry, and applied physics with the goal of producing two specific optical devices. Of relevance to our work, she points out that mathematics can serve as a communication channel between disparate scientific fields. A particular equation or formula may have at least some shared meaning across many disciplines, and scientists can set up "dictionaries" relating the terminologies of their fields around this formula. She distinguishes a "speaker's dictionary" from a "listener's dictionary" in this regard. Bracken and Oughton (2006) are outside the canonical STEM disciplines but highlight the useful concepts of dialect and articulation, which became central to our work: "Dialects represent the difference between the everyday use of a word and expert use and the ways in which different disciplines use the same word to mean different things" (p. 376), whereas articulation "involves deconstructing one's own disciplinary knowledge in conjunction with those of other disciplines in order to understand the building blocks and thereby reconstruct a common understanding" (pp. 377-378). In 
the realm of educational collaborations, Donaldson, Felzian, Marvin, Cielocha, and Shapiro (2019) developed a common instructional treatment of energy conservation across the disciplines of physics, biology, chemistry, and biochemistry via a shared "Energy Diagram" representation. They note that they "meet regularly and visit each other's classes in order to best integrate our disciplines" (p. 533). Like our own group, they "discover our own misconceptions as they apply to other disciplines and work together to correct them" (p. 533). Redish and Cooke (2013), a physicist and biologist, respectively, describe their collaboration focused on developing an introductory physics course for biology students. For such a purpose, they observe that physicists and biologists need to "develop an understanding of the other's discipline, not just the content but also the epistemological style and goals" (p. 185). There are also collaborative efforts between biology and mathematics aimed at incorporating more biology examples in mathematics courses and more quantitative reasoning in biology courses (Marsteller, 2010). Finally, Diaz Eaton et al. (2019) discuss the various meanings of model and modeling in educational contexts across mathematics, statistics, and biology. They suggest that "if both mathematicians and biologists appreciate that the same model given with formulas by the mathematician can be thought of in terms of graphs, data, or experiences by the biologist, then it is much easier to achieve a common understanding that is more nuanced than the individual understandings of the members of each discipline" (p. 803).

The concept of trading zones introduced to science studies by Galison (2010) describes a shared language that develops at the interface between scientific fields to facilitate collaboration between their practitioners on a joint project. Often this involves a simplification of the language of both fields to a common (or new) core that is sufficiently well understood by both for the practical purposes at hand. This construct seems a better fit for scientific than educational collaborations, however. Our group did learn to communicate across disciplinary boundaries, but we also critically examined the assumptions behind our various languages and the challenges they raise for student learning. The goal was to share deep features of our own languages rather than to create a new one.

In writing this paper, we began by summarizing both individually and collectively the most salient crossdisciplinary issues from our earlier discussions: those that we returned to multiple times, those that we found most difficult to communicate clearly about, those that led us to unexpected views about our own discipline or others, and those that gave us insight into our students' struggles or our own teaching. We held additional conversations about these to explore how (and if) we had resolved them, how our thinking may have changed in retrospect, and what lessons they might provide for other instructors or cross-disciplinary collaborations. We sought out discussions of similar issues in the research literature and their treatment in textbooks. Ultimately, the conclusions we present are based on our own opinions and experiences during this collaboration. Unsourced statements herein that sound dogmatic ("Chemists think...", "Mathematicians are...") should be interpreted as our own views.

Four main themes emerged from our discussions and will be illustrated in the examples that follow.

1. People in different disciplines use the same words to mean different things. This is the meaning of dialect as introduced above, and we spent a lot of time engaged in translation. It may be obvious that a solution is not the same thing for a chemist as for a mathematician, but as we found, a function is not the same thing either.

2. People in different disciplines may use different words to express the same thing. This and the previous point can be counterintuitive in view of the fact that technical terminology is usually introduced to avoid ambiguity. However, its ability to do this depends on what range of alternative meanings needs to be avoided, and that can vary from one discipline to another. Our efforts to understand one another's terminology and explain our own exemplified articulation as defined above.

3. Much implicit information underlies our disciplinary understanding. Scientists know from experience when particular approximations are reasonable and when particular mathematical models apply. They know what terminology is applicable and standard in their field even when it is ambiguous or not literally accurate.

4. Scientists intuitively think about mathematics in the context of real-world applications. They begin with data, and for them, mathematics is a tool, not the main object of attention. Mathematical abstraction is often irrelevant to scientists, for whom the use of this tool is grounded in concrete experimental data in a specific applied context. The tool just needs to function reliably in that context.

We will follow these themes through a series of topics that arose in our discussions.

\section{Topics of Discussion}

\section{Functions}

Functions are of central importance in mathematics. They are the main tools for representing and analyzing 
covariation of quantities in the real world as well as structural relationships within mathematics itself. Covariation of quantities in the real world is at the heart of science, but in contrast to mathematics, in science it is the quantities themselves (population, energy, chemical concentration, etc.) that tend to be emphasized. Many of our conversations revolved around this difference in viewpoint.

The differential equations video scripted by a mathematician in our group presents a differential equation as analogous to an algebraic equation: the problem is to find an unknown object given some information about it. The notation emphasizes that the unknown object is a function rather than a number. Solving the equation means finding that function (if initial conditions are given) or all possible such functions more generally. Mathematicians tend to use the Lagrange notation for derivatives to convey this, as in $f^{\prime}(x)=r f(x)$, where $f$ is the name of the function being sought. The scientists in our group were more comfortable with Leibniz notation, such as $\frac{d N}{d t}=r N$, reflecting the viewpoint that it is the value of the quantity $N$ at time $t$ that is sought. The disciplinary difference makes sense given that quantities in science such as energy are tangible and measurable, whereas the generic variables $x$ and $y$ connected by a function in mathematics have no fixed meanings and only their covariation is of interest. Thus, a scientist will denote energy as $E$ regardless of what other quantities it may depend on, whereas a mathematician is likely to give distinct names to the various functions that express energy in terms of different possible independent variables. Scientists name quantities, mathematicians name functions. Perhaps the ultimate expression of this difference is in thermodynamics, much of which consists of relating the partial derivatives of such quantities with respect to one another, using Leibniz notation.

The mathematicians in our group conceptualized a chemical "rate law" as a differential equation, and the corresponding "integrated rate law" as a solution to that differential equation, specified by a particular initial condition. This viewpoint was understood by, but did not resonate with, the chemists. To a chemist, a rate law connects concentration to reaction rate, while an integrated rate law connects concentration to time. The former provides information about an underlying reaction mechanism (first order, second order, etc.), while the latter describes a particular realization of that mechanism over time in a specific experiment. Both are useful ways to summarize experimental data. Neither one is a "problem" to which the other is a "solution". The chemists would not use the term solution in this way, but rather for a numerical solution to an algebraic equation (or for a liquid chemical mixture!). The mathematicians viewed the initial concentration as an unknown or arbitrary constant $C$, whereas for the chemists this is known and part of a specific experimental protocol. The distinction may seem minor but we can attest that it did impede our communication.

We found it challenging to agree on what function means in a scientific context. Is a table or graph of discrete data points a function? Is a smooth curve fit to that data a function? Or is it a specific functional form, say exponential, that satisfies a differential equation coming from a plausible model for the data? The mathematicians tended to say that these were all functions, but then which is the "real one" that actually represents the quantity or relationship under study? This is a question of mathematical modeling whose subtlety is often concealed from students. In physics, it might be natural to claim that a thrown ball has a real trajectory given by some true function (quadratic in the absence of drag forces), which our measurements would reveal if not for small effects of "noise," but is there a similar "true" function giving the growing population of seals on an island, as distinct from one of many functions we might fit to that data? The scientists were most likely to apply the term function to an analytic formula resulting from explicitly solving a differential equation or fitting a curve to data. For them, data are primary, a function is part of a model.

The use of continuous variables and functions to model processes that are fundamentally discrete (such as population growth over time) does occur in our videos, but we do not discuss any formal justification for this practice. This is partly due to time constraints, partly to this issue being peripheral to those we wanted to address, but also to the practical attitude of the scientists that one knows when this is a sufficiently good approximation for the purposes at hand. Our biologist made the point that real data are always sampled at discrete times, and measurement and modeling errors are likely to be more significant than those introduced by smoothing the data to look continuous.

The mathematicians often spoke of differentiation or integration as operations performed on functions, producing other functions as their results. The scientists might think of performing operations on graphs, but usually thought of the results in terms of quantities such as the slope at a point or the area under the graph, which might have concrete meanings in any specific context, perhaps the velocity of an object or a count of biological molecules.

\section{Rate constants}

A frequently recurring issue in our discussions was the meaning of rate constants characterizing exponential 
growth or decay processes. We discussed this in the contexts of elementary differential equations (mathematics), population growth (biology), and chemical kinetics (chemistry). It also arises in radioactive decay (physics), but this was not a subject of our videos. The differential equation for exponential growth is

$$
\frac{d N}{d t}=r N
$$

where $r>0$ for growth and $r<0$ for decay. Here, $N$ may be a number of organisms or atoms, or a chemical concentration. The solution is $N(t)=N_{0} e^{r t}$, where $N_{0}$ is the amount at time zero. The differential equation is often derived from the change during a finite time interval, expressed as $\Delta N=r N \Delta t$, by taking the limit as $\Delta t \rightarrow 0$. The doubling time (or half-life, in the case of decay) is $(\ln 2) /|r|$. The underlying modeling assumption that the growth rate is proportional to the amount present would be called a first-order reaction in chemistry, or an exponential population growth model in biology.

The questions began with the proper description of the rate constant $r$, which has units of $1 /$ time. Is it the fraction of $N$ that is added/removed per unit time, as its units and the finite-time equation would suggest? If so, then in the case $r=1$, the amount should double in a unit time, since $100 \%$ is added, but this contradicts both the formula for doubling time and the explicit solution, the latter predicting growth by the larger factor of $e \approx$ 2.718. Similarly, if $r=-1$, then the full amount should be gone at $t=1$, but this does not occur for any finite time in the explicit solution. As a less dramatic example, suppose we measure $N(0)=100$ and $N(1)=120$, so that the growth in unit time is $20 \%$. If we conclude that $r=$ 0.2 and $N_{0}=100$, then the explicit solution predicts $N(1)=122$, contradicting our original measurement!

Our experience is that textbooks rarely point out these potential discrepancies or give students opportunities to think through them. The mathematicians in our group saw this as a quintessential example of the crucial distinction between an average and an instantaneous rate of change. The $20 \%$ growth measured between times 0 and 1 is an average rate of change that must be distinguished from the instantaneous rate of change at any given time, which according to the differential equation is equal to the value of $r N$ at that time. Thus, the value of $r$ in the differential equation cannot be (immediately) determined from such a measurement. The two constants denoted $r$ in the differential equation and in the finite-time equation are in fact not the same and should not be confused. The finite-time equation cannot be used to determine the constant in the differential equation except approximately in the case that $\Delta t$ is very small, $\Delta t \ll 1$. Sometimes textbooks recognize this, as in Urry et al. (2014), where the finite-time parameter is called $r$ while the differential equation parameter is called $r_{\text {inst }}$, but with no explanation of how these might be related. However, in Campbell and Reece (2005), which is a primary source for Urry et al., they are both simply $r$. Neither textbook provides the explicit solution to the differential equation, so that students cannot explore its numerical predictions as above.

Interestingly, in more recent editions of both textbooks (Urry, Cain, Wasserman, Minorsky, \& Reece, 2017; Urry, Wasserman, Minorsky, Jackson, \& Reece, 2016), the distinction between the two rate constants is clearly made. The constant in the finite-time equation, now called $r_{\Delta t}$, is accurately described as the per capita growth rate during the time interval $\Delta t$ and is distinguished from the constant $r$ in the differential equation. It is explained that they become more nearly equal as the interval $\Delta t$ gets smaller. However, the description of $r$ as "the per capita change in population size that occurs at each instant in time" (Urry et al., 2016, p. 858) will still leave students puzzled as to how a continuously growing population can change at a single instant.

Describing $r$ (the constant in the differential equation) as the fractional change per unit time is thus misleading (although standard) unless it is emphasized that this is an instantaneous rate of change that cannot literally be applied to a "unit time" but only to much smaller time intervals. A helpful analogy from everyday life is the difference between simple and compound interest. A 20\% annual simple interest rate (unrealistic as it may be) means that your money grows by $20 \%$ over 1 year: it is an average rate of change. An annual interest rate of $20 \%$ that is compounded daily (or ideally, continuously) means your money grows by $0.2 \Delta t$ in each short time interval $\Delta t=1 / 365$ years. This is (much closer to) an instantaneous rate of change and is equivalent to the larger simple interest rate of about $22 \%$ per year as pointed out above.

Initially, the group members with chemistry and biology backgrounds were confused by the mathematicians' concerns about confounding the two rate constants, perhaps because average rates of change are all one can measure, or because relevant growth rates are usually small enough that the difference is unimportant. Implicit disciplinary knowledge tells them which type of rate is meaningful. The compound interest example was readily understood by the whole group and served as a conceptual stepping stone that, with further conversation, helped the mathematicians come up with an alternative example that made the case in a discipline-relevant way (because it resembles the integrated rate law for a firstorder chemical reaction). Taking the logarithm of the explicit solution gives $\ln N=\ln N_{0}+r t$. This shows that a 
semilog plot of $N$ versus time will be a straight line of slope $r$, so $r$ is correctly described as the change in $\ln N$ per unit time. Indeed, the differential equation itself can be rewritten in the form $\frac{d \ln N}{d t}=r$, confirming that $\ln N$ changes at a constant rate. We think that students would derive a more solid understanding of rates of change and of exponential growth from working through such a sequence of puzzles and explanations. One moral of our discussion is that the canonical explanations provided in one discipline may not mesh with the typical viewpoints of another. For example, semilog plots are more familiar tools in science than they are in mathematics. A second lesson is that it is helpful to have more than one representative of each discipline in the discussion. Different mathematicians in our group generated the various examples discussed in this section. "Two people can bring different perspectives and if one explanation flounders, then the second person can draw on alternative language and examples to assist cross-disciplinary understanding" (Bracken \& Oughton, 2006, p. 380). For the same reason, individual participants with cross-disciplinary backgrounds can facilitate understanding. One mathematician in our group had a physics background and was able to help the mathematicians and scientists translate for one another.

\section{Units}

Scientists work with quantities that are actually measured and typically have associated units, e.g., meters per second, or calories. These units are included in the stated values of the quantities, e.g., $E=10$ Joules. Equations expressing scientific laws are required to be dimensionally consistent, and the units are included when quantities are substituted into them. The units of an unfamiliar quantity often provide insight into its meaning. Mathematicians typically work with pure numbers, however. In modeling, they may agree that all lengths will be expressed in meters, but then simply write $l=6$. Indeed, it was considered a great advance in mathematical notation when it became possible to write, for example, $y=$ $x^{2}+x+1$ without worry that this is meaningless because $x$ must represent a length and $x^{2}$ an area, which cannot be added together. In one of our physics videos, the similar kinematic equation $x=a t^{2}+b t+c$ is analyzed dimensionally to show that $b$ has units of velocity and $a$ has units of acceleration, an important clue to their physical meanings. We think that explicit crossdisciplinary comparisons of this type would be very helpful for students.

The area under a curve (graph) plays a role in some of our videos, and the scientific meaning of this area can often be guessed from its units (the product of the units on the separate axes). For example, the area under a graph of force versus distance represents work or energy. The scientists tended to reach an interpretation of such an area based primarily on its units, while the mathematicians preferred to base such an interpretation on the meaning of the individual terms in a Riemann sum approximation. We were all puzzled by an example from pharmacokinetics, where the area under a graph of the bloodstream concentration of some drug versus time is assumed to represent the amount of the drug absorbed by the body and biochemically active there (Urso, Blardi, \& Giorgi, 2002). Although this is plausible, we could not justify it in either fashion. The units of gram-second/ milliliter do not immediately translate into a total amount (grams?), and Riemann-sum explanations are also elusive in the literature we examined. Sometimes "arbitrary units" or a.u. appear on the axes of graphs (Beal et al., 2018), which still allows one to compare the relative areas of two regions if not their absolute magnitudes. In some cases, the quantities measured and plotted are proxies for the quantities of actual interest and may not share their units at all. Implicit disciplinary knowledge tells practitioners in the field that such proxies are adequate for the purpose at hand, while those in other disciplines expressed confusion. For example, in one of our biology videos, the measured fluorescence of a fluorescent dye is a proxy for the amount of dye bound to DNA, which is a proxy for the amount of DNA itself. The relative areas under portions of the corresponding graph indicate relative numbers of distinct cell populations. This kind of proportional reasoning is likely to be unfamiliar to students. Of course, without explicit instruction, students are unlikely to appreciate when quantities are serving as proxies for others, and especially that such proxies are only adequate under certain experimental conditions (Beal et al., 2018).

Since it is rare to have an explicit algebraic equation for an experimentally measured graph, scientists often resort to numerical methods to estimate areas under such curves (or, in the old days, cutting out and weighing the region from graph paper). This has led to amusing results when the scientific literature has reinvented the wheel in the form of methods like the trapezoidal rule that are standard in calculus courses (Tai, 1994). This points to another benefit of increasing the crossdisciplinary knowledge of STEM faculty.

\section{Sign conventions}

The sign of a rate of change is important, conveying the information as to whether the changing quantity is increasing or decreasing. The sign may be an arbitrary convention, or result from a deliberate choice of coordinates, for example, when the positive $z$ axis is chosen to point upward from the Earth's surface, so that an object moving upward will have a positive velocity, rather than 
the reverse. When a convention becomes universal within a discipline, it may be viewed as necessary rather than conventional by students and teachers alike. Some of our discussions concerned chemical reaction rates, which are conventionally positive in chemistry even though they may be measured by the rate of appearance of one chemical or the rate of disappearance of another. This means that an explicit minus sign might appear in a formula relating a reaction rate to some derivative of concentration. This can be problematic for students (Lamb et al., 2012), who may assume that an explicit minus sign as in $-x$ always denotes a negative number rather than one having the opposite sign to that of $x$. A similar convention occurs in physics when speed, always positive, is defined as the absolute value of velocity, which may have either sign. In chemistry, the concept of absolute value is not usually invoked to explain the rate convention. Our discussions suggested that the explicit use of absolute value to define reaction rate might be pedagogically beneficial.

Potentially confusing sign conventions are ubiquitous in the STEM curriculum. Examples include heat flow in thermodynamics (Is it positive when flowing into or out of the system? Does positive heat flow raise or lower temperature?), acceleration (Is it the rate of change of velocity, as usual in physics, or of speed, as usual in everyday life, where deceleration means slowing down?), velocities of stars and galaxies (Does positive redshift indicate motion toward or away from the observer?), and electric current (Thanks to Ben Franklin, a positive current is carried by negatively charged electrons flowing in the opposite direction). In mathematics, -10 is a smaller number than -3 despite having a larger magnitude. Chemical $\mathrm{pH}$ is defined with an explicit minus sign to ensure that it is positive and hopefully avoid such confusion.

\section{Terminology}

We encountered many examples of different disciplines using the same words to mean different things, or different words to express the same meaning, including the following.

In calculus courses, the distinction between the shapes of the parabolas $y=x^{2}$ and $y=-x^{2}$ is always expressed by saying that the former is concave up and the latter concave down (that is, the concave side of the curve is facing up or down). In more advanced mathematics, the term convex is often used in place of "concave up," but never at first-year level. Indeed, convex has other meanings as in a convex polygon, or lens. The scientists in our group were familiar with concave and convex lenses and shapes, but did not use "concave up" or "concave down" to describe graphs, and found the mathematicians' use of these terms unintuitive. One referred instead to positive or negative curvature, which would be understood differently in mathematics. One described the second graph as an "upside-down parabola" although in mathematics a parabola may have any orientation in a plane.

The scientists sometimes described a functional relationship $y=a x+b$ by saying that $y$ is linearly dependent on $x$. The mathematicians were reluctant to use this term outside the subject area of linear algebra, although both groups were happy to call the relationship proportional if $b=0$.

The mathematicians sometimes spoke of the input to a function, whereas the scientists would think of the independent variable, the one being experimentally controlled.

Scientists sometimes used the phrase "change over time" to describe an instantaneous rate of change (derivative). Mathematicians preferred "rate of change with respect to time" on the grounds that "change over time" is ambiguous between average and instantaneous rates, or could even mean the total amount of change; and that "over" is ambiguous between "during (a time interval)" and "divided by (the duration of the interval)". Similarly, some of us have encountered students who interpret the "over" in "integral of a quantity over time" as indicating division.

The scientists observed that mathematicians sometimes used the terms "integral" and "antiderivative" interchangeably, and sometimes as quite distinct. "Antiderivative" was not commonly used by the scientists, at least since their own first-year calculus courses, and indeed may be rare outside calculus textbooks. An antiderivative of a function $g(x)$ is any function whose derivative is $g$; the indefinite integral $\int g(x) d x$ denotes all such functions, which is why it includes an arbitrary constant $C$. However, "integral" more often means the definite integral, $\int_{a}^{b} g(x) d x$, which is defined as the limit of a Riemann sum and gives the area under the graph of $g$ between specific vertical lines $x=a$ and $x=b$. There is no a priori relationship between this and an antiderivative; the importance of the Fundamental Theorem of Calculus (FTC) is precisely that it establishes such a relationship and allows areas to be computed from antiderivatives. Students who conflate the two types of integral - and many do-often do not appreciate the significance of the FTC (Wagner, 2018).

\section{Notation}

The notation used in a discipline is a part of its language, raising the same issues of dialect and articulation as does terminology. Notations adopted within a field often reflect compromises between making the meaning as unambiguous as possible (prevalent in mathematics) and making computations as easy as possible (prevalent 
in science). Notational differences we tried to reconcile in our discussions included the following.

In chemistry and biology texts, it is not uncommon for the notations $d x$ and $\Delta x$ to be used interchangeably, which risks confusion between average and instantaneous rates of change (Seethaler et al., 2018).

Physicists often omit the limits of integration from a definite integral when these are thought to be clear from the context, for example, $\int F d t$ may mean $\int_{-\infty}^{\infty} F d t, \int_{0}^{\infty}$ $F d t$, or even $\int_{0}^{t} F d t$. In a calculus text, the absence of limits would indicate an indefinite integral.

In addition to the forms already mentioned for the differential equation of exponential growth, some chemistry/biology sources give a differential form such as $N_{t+d t}-N_{t}=r N_{t} d t$. This would not be standard in mathematics at calculus level.

\section{Is physics different?}

Thus far, we have included the physicist in our group along with the chemists and biologist as "scientists," in contrast to the mathematicians among us. One might surmise, though, that he had fewer communication issues with the mathematicians than did the other scientists, and to some extent, this was true. Calculus, after all, arose in conjunction with Newtonian physics and shares some concepts, notation, and paradigmatic examples (such as the instantaneous velocities and accelerations of particles moving under the influence of forces like gravity) with it. Still, much has been written about the cultural and pedagogical differences between abstract mathematics and its more concrete applications in physics (Dray et al., 2019; Redish \& Kuo, 2015). Examples that came up in our discussions included the use of infinitesimal differentials such as $d x$, which is encouraged for physics students but discouraged for mathematics students (Dray et al., 2019; Lopez-Gay, Martinez Saez, \& Martinez Torregrosa, 2015); the focus on quantities as opposed to functions; and the reliance on units and dimensional analysis for checking equations as well as generating possible relationships between quantities. We also noted the reversal of the meanings of the two angular spherical coordinates $\theta$ and $\phi$ between the physics literature and many calculus textbooks (Dray \& Manogue, 2003), and the frequent absence from calculus syllabi of the spherical coordinate unit vectors $\hat{\rho}, \hat{\theta}, \hat{\phi}$ commonly used in physics.

\section{Is mathematics different?}

The "practical" mathematics used by scientists differs in significant ways from the "abstract" or "structural" mathematics taught by mathematicians. Scientists in our group said that they had actually learned this practical mathematics from their disciplinary science teachers and not connected it to prior mathematics courses. The scientists were often impatient with the mathematicians' obsession with technical correctness and with extreme or pathological cases that would never arise in practice. They sometimes found mathematicians' thinking too abstract, context-free, or ungrounded in reality. One of our videos discussed a problem about the rate of change of the volume of fluid in a tank, and we debated how much context a student viewer might need for the type of tank in question. The mathematicians were quite content with an abstract container having no particular shape or function. They were also willing to picture it as a hot water tank or toilet tank. In contrast, the scientists were not satisfied with a generic example or one that would not realistically empty and fill in the erratic manner described in the problem context. We ultimately decided to ground this in the relatable context of a rain barrel, which might plausibly fill and empty in roughly the manner modeled in the video.

The foundations and justifications of mathematical techniques, which are given center stage in mathematics courses, may be taken for granted in science, where practical knowledge determines what is reasonable to do and to assume. The scientists in our group came to value these foundational issues (to some extent), and the mathematicians gained an understanding of the other resources scientists can draw on. For example, proof is the standard of correctness in mathematics, but in science, experimental data have the final word. Mathematics is merely a tool for many scientists, who may see the mathematician's role as maintaining the tool and certifying that it is in working order.

\section{Conclusions}

Designing videos that present a concept from multiple disciplinary viewpoints, to be viewed by students in multiple disciplinary programs, was challenging. In writing and revising the scripts, we needed to be aware of discipline-specific terminology and notation that might be misunderstood and implicit assumptions that needed to be spelled out. We realized that students in different fields might attend to different aspects of the videos than we intended or indeed focus on features we considered irrelevant and had overlooked. It took time (far more than we anticipated) to explain and appreciate our various disciplinary cultures and expectations for students and to agree on syntheses faithful to each discipline but understandable across them. Learning multiple languages for cross-disciplinary communication increased our empathy and respect for our students, who have the same experience as they move from one STEM classroom to another in the course of their studies.

Most of the issues we discussed arose multiple times in multiple contexts. This allowed us to revisit them 
from different perspectives and extend and deepen earlier discussions. We shared our disciplinary perspectives and tried to make our implicit knowledge as explicit as possible. It was important that we created a climate of mutual respect that allowed us to ask even very elementary questions comfortably. One technique that facilitated cross-disciplinary understanding for us was to instantiate the calculus concepts in contexts that were broadly shared and not discipline-specific. Compound interest was one such context, and the motion of vehicles or projectiles was another. We could communicate and reconcile our ideas using that shared framing and subsequently translate our understanding into situations specific to our own disciplines.

The in-depth discussions we engaged in were necessary to the success of our project, but we found them surprisingly valuable for their own sake. Recalling the four themes laid out in the "Introduction" section, we have described multiple examples of the varied meanings of terminology and notation across fields, the use of implicit disciplinary knowledge, and tensions between pure and applied mathematics. Basic concepts such as functions, rates, and units are understood differently across disciplines, and there is a gap between what is emphasized in mathematics and in the sciences. Many STEM instructors have not had opportunities to reflect on these differences themselves, a precondition for communicating them to their students. We strongly recommend that faculty take the time to engage in similar crossdisciplinary discussions and apply the resulting insights to their own teaching.

Our advice to other educationally focused STEM collaborations overlaps that of Diaz Eaton et al. (2019). Have a shared goal, and a concrete deliverable (the videos in our case). Be willing to act as both a student and a teacher, that is, both an expert in your own field and a novice in others. Be explicit about language, anticipating that familiar terminology may have another meaning in another discipline and may be understood within a different conceptual context. Seek out common concepts that are expressed differently across disciplines. Be open to appreciating why another discipline might do familiar things differently and look critically at your own. Redish and Cooke (2013) recommend "respect for each other's discipline and insights; a willingness to reconsider one's own discipline from a different point of view; and finally, patience, persistence, and humor" (p. 185).

\section{Acknowledgements}

This paper is dedicated to the memory of Jeffrey Remmel, former PI of our project.

\section{Authors' contributions}

JMR was the primary author of the manuscript. All authors participated in the discussions reported herein. The authors read, edited, and approved the manuscript.
Funding

This work was supported by NSF grant IUSE 1610193.

Availability of data and materials

Not applicable.

\section{Competing interests}

The authors declare that we have no competing interests.

\section{Author details}

'Department of Mathematics, University of California San Diego, La Jolla, CA, USA. ${ }^{2}$ Department of Physics, University of California San Diego, La Jolla, CA, USA. ${ }^{3}$ Department of Chemistry and Biochemistry, University of California San Diego, La Jolla, CA, USA. ${ }^{4}$ Section of Cell and Developmental Biology, University of California San Diego, La Jolla, CA, USA. ${ }^{5}$ Division of Physical Sciences, University of California San Diego, La Jolla, CA, USA.

Received: 3 July 2020 Accepted: 21 December 2020

Published online: 19 February 2021

\section{References}

Bain, K., \& Towns, M. H. (2016). A review of research on the teaching and learning of chemical kinetics. Chemical Education Research and Practice, 17(2), 246262

Beal, J., Haddock-Angelli, T., Baldwin, G., Gershater, M., Dwijayanti, A., Storch, M. . with the iGEM Interlab Study Contributors (2018). Quantification of bacterial fluorescence using independent calibrants. PLoS One, 13(6), e0199432.

Bracken, L. J., \& Oughton, E. A. (2006). "What do you mean?" the importance of language in developing interdisciplinary research. Transactions of the Institute of British Geographers, 31(3), 371-382.

Campbell, N. A., \& Reece, J. B. (2005). Biology, (7th ed., p. 1144). New York City: Pearson.

Diaz Eaton, C., Highlander, H. C., Dahlquist, K. D., Ledder, G., LaMar, M. D., \& Schugart, R. C. (2019). A "rule-of-five" framework for models and modeling to unify mathematicians and biologists and improve student learning. PRIMUS, 29(8), 799-829.

Donaldson, N. L., Felzian, L. K., Marvin, M. C., Cielocha, J. J., \& Shapiro, T. (2019). Development of an interdisciplinary conceptual conservation of energy theme for use in undergraduate physics, chemistry, and biology courses. American Journal of Physics, 87(7), 527-534.

Dray, T., Gire, E., Kustusch, M. B., Manogue, C. A., \& Roundy, D. (2019). Interpreting derivatives. PRIMUS, 29(8), 830-850.

Dray, T., \& Manogue, C. (2003). Spherical coordinates. The College Mathematics Journal, 34(2), 168-169.

Duncker, E. (2001). Symbolic communication in multidisciplinary cooperations. Science, Technology \& Human Values, 26(3), 349-386.

Galison, P. (2010). Trading with the enemy. In M. E. Gorman (Ed.), Trading zones and interactional expertise. Cambridge: MIT Press.

Herbert, S., \& Pierce, R. (2011). What is rate? Does context or representation matter? Mathematics Education Research Journal, 23(4), 455-477.

Jones, S. R. (2017). An exploratory study on student understandings of derivatives in real-world, non-kinematics contexts. The Journal of Mathematical Behavior, $45,95-110$.

Jones, S. R. (2019). What education research related to calculus derivatives and integrals implies for chemistry instruction and learning. In M. H. Towns, K. Bain, \& J.-M. G. Rodriguez (Eds.), It's all just math: Research on students' understanding of chemistry and mathematics, ACS symposium series (pp. 187212). Washington D.C: American Chemical Society 2020.

Lamb, L. L., Bishop, J. P., Philipps, R. A., Schappelle, B. P., Whitacre, I., \& Lewis, M. (2012). Developing symbol sense for the minus sign. Mathematics Teaching in the Middle School, 18(1), 5-9.

Lopez-Gay, R., Martinez Saez, J., \& Martinez Torregrosa, J. (2015). Obstacles to mathematization in physics: The case of the differential. Science \& Education, 24(5-6), 591-613.

Marsteller, P. (2010). Beyond BIO2010: Integrating biology and mathematics: Collaborations, challenges, and opportunities. CBE Life Sciences Education, 9, $141-142$.

Redish, E. F., \& Cooke, T. J. (2013). Learning each other's ropes: Negotiating interdisciplinary authenticity. CBE Life Sciences Education, 12, 175-186. 
Redish, E. F., \& Kuo, E. (2015). Language of physics, language of math: Disciplinary culture and dynamic epistemology. Science \& Education, 24, 561-590.

Rodriguez, J., Bain, K., Towns, M. H., Elmgren, M., \& Ho, F. (2018). Covariational reasoning and mathematical narratives: Investigating students' understanding of graphs in chemical kinetics. Chemical Education Research and Practice, 20, 107-119.

Seethaler, S., Burgasser, A., Bussey, T. J., Eggers, J., Lo, S. M., Rabin, J. M., ... Weizman, H. (2020). A research-based checklist for development and critique of STEM instructional videos. Journal of College Science Teaching, 50(1), 30-36.

Seethaler, S., Czworkowski, J., \& Wynn, L. (2018). Analyzing general chemistry texts' treatment of rates of change concepts in reaction kinetics reveals missing conceptual links. Journal of Chemical Education, 95, 28-36.

Tai, M. M. (1994). A mathematical model for the determination of total area under glucose tolerance and other metabolic curves. Diabetes Care, 17(2), 152-154 See also the letters commenting on this article, and the author's response, in Diabetes Care, 17(10), 1223-1227.

Thompson, P. W. (1994a). Images of rate and operational understanding of the fundamental theorem of calculus. Educational Studies in Mathematics, 26, 229-274.

Thompson, P. W. (1994b). Students, functions, and the undergraduate curriculum. In E. Dubinsky, A. H. Schoenfeld, \& J. J. Kaput (Eds.), Research in Collegiate Mathematics Education 1 (issues in mathematics education Vol. 4), (pp. 21-44). Providence: AMS.

Thompson, P. W., Byerley, C., \& Hatfield, N. (2013). A conceptual approach to calculus made possible by technology. Computers in the Schools, 30, 124-147.

Thompson, P. W. \& Carlson, M. P. (2017). Variation, covariation, and functions: Foundational ways of thinking mathematically. In J. Cai (Ed.), Compendium for research in mathematics education, (pp. 421-456). Reston: NCTM.

Thompson, P. W., \& Silverman, J. (2008). The concept of accumulation in calculus. In M. P. Carlson, \& C. Rasmussen (Eds.), Making the connection: Research and teaching in undergraduate mathematics education, (pp. 43-52). Washington D. C: MAA.

Urry, L. A., Cain, M. L., Wasserman, S. A., Minorsky, P. V., Jackson, R. B., \& Reece, J. B. (2014). Campbell biology in focus, (p. 836). New York City: Pearson.

Urry, L. A., Cain, M. L., Wasserman, S. A., Minorsky, P. V., \& Reece, J. B. (2017). Campbell biology, (11th ed.). New York City: Pearson.

Urry, L.A., Cain, M.L, Wasserman, S.A., Minorsky, P.V., Jackson, R.B., \& Reece, J.B. (2016). Campbell biology in focus $2^{\text {nd }}$ edition,. New York City: Pearson.

Urso, R., Blardi, P., \& Giorgi, G. (2002). A short introduction to pharmacokinetics. European Review for Medical and Pharmacological Sciences, 6, 33-44.

Wagner, J. F. (2018). Students' obstacles to using Riemann sum interpretations of the definite integral. International Journal of Research in Undergraduate Mathematics Education, 4, 327-356.

\section{Publisher's Note}

Springer Nature remains neutral with regard to jurisdictional claims in published maps and institutional affiliations.

\section{Submit your manuscript to a SpringerOpen ${ }^{\circ}$ journal and benefit from:}

- Convenient online submission

- Rigorous peer review

- Open access: articles freely available online

- High visibility within the field

- Retaining the copyright to your article

Submit your next manuscript at $\boldsymbol{\nabla}$ springeropen.com 